\title{
The morphological features and properties of some soils south west paris oasis, Egypt
}

\author{
M.E.M. Wahdan, M. Niazy. And A. M .A .Zayed. \\ Soils Water and Environment research Institute, Agricultural Research Center, and Giza, Egypt. \\ Corresponding author: mostafawahdan58@gmail.com
}

\begin{abstract}
The study area is located south west of Paris Oasis and occupies about 178218 hectare and represented by 29 profiles. The study are which had rock formation belong to Cretaceous Nubian Sandstones, and has four landform units:

1- Sand sheet: These soils cover about 95042 hectares representing 53.33\% of the total studied area and are classified according to USDA (2014) to seven taxonomic units Coarse loamy, mixed, hyperthermic. TypicTorriorthents

a- Fine loamy, mixed, hyperthermic. TypicTorriorthents. b- Sandy, mixed, hyperthermic.TypicTorriorthents. c-Sandyover loamy,mixed,hyperthermic.TypiTcorriorthents.d-fine loamy ,mixed, hyperthermic .Lithic Torriorthents e - Sandy, mixed,hyperthermic.LithicHaplogypsids.f-Sandy, siliceous, hyperthermic. Typic Torripsamments,

2-Sabkha: These soils covera bout 3701 hectares representig2.07\% of the total studied area and are classified to family levelas. a- GypsicHaplosalids, f i n e clayey,mixed,hyperthermic. b- TypicHaplosalids,sandy, mixed, hyperthermic.

3-Pediplain: The soils under consideration have area of about 63428 hectare and represent $35.60 \%$ of the total area.The soils of this unit are classified as follows: a- Sandy,mixed,hyperthermic.TypicTorriorthents. b-Fine, loamy , mixed, hyperthermicLithic Torriorthents. c-Coarse loamy, mixed,hyperthermic. Lithic Torriorthents.

d- Coarse loamy, mixed,hyperthermic.TypicTorriorthents.

4-Barchan dunes belts.

Soils of dunes occupy about 16047 hectare and represent about 9.00\% of total area.Theyhave one taxonomic unit: a-- sandy, siliceous,hyperthermic TypicTorripsamment.

-Land suitability evaluation

Values of suitability indices for the current suitability are marginally class (S3) except soils of profiles3, 5and25 which a r e not suitable(N1), soils of profiles 28 and29which are not suitable(N2), and Soils of profiles1, 15, 17,26 and27which are moderately suitable (S2) On the other hand, these soils would be moderately suitable (S2) their potential condition. Soils of profiles 17,24and26 which would be highly suitable(S1), and soils of profiles2,3,4,5,6,79,12,18and19 which would be Margin( S3). The soils of profiles 28 and 29 are permanently not suitable (N2).
\end{abstract}

Key words: Sabkha, Pediplain, Barchan dunes belts, Land Form, Land classification, Land evaluation

\section{Introduction}

Increment of population and limited land in the Nile River valley and delta put stress on the government to create and establish strategic plans for horizontal expansion in the western Desert for food security. The study area is considered one of the promising areas of horizontal expansion in the western Desert. The reclamation of this area aims at establishing channel system between the south Valley project and Al-Kharga Oasis and developing the areas around Darb El-Arabaein roadas well (AbdelKawy and Darwish, (2014).

The study area has rock formation belong to Cretaceous Nubian Sandstone according to Atlas of $\operatorname{Egypt}(\mathbf{1 9 2 8})$.The rock land is less high, slopes are less steep, gullies less narrow and there are considerable wind-blown sand accumulations in the gullies and lower parts in general
(FAO/SF:16/UAR,1963). The geological map, which produced by EGSA(1988), reveals to the sand sheets formation cover the eastern part and have the predominant area, followed by Sabaya formation(Desert Rose Beds) and sand dunes are concentrated in the western part, while sabkha deposits cover small parts in the north east of the study area.

The climatological data (Table1) indicate that the highest temperature value was recorded in August $\left(44.1^{\circ} \mathrm{C}\right)$ and the lowerst one was recorded in December $\left(24.3^{\circ} \mathrm{C}\right)$. The total rainfall was 0.20 $\mathrm{mm} /$ year. Relative humidity ranged between 14 and $38 \%$.Averages of evaporation, wind speed and sun shine were $7.76 \mathrm{~m} \backslash$ month, $2.7 \mathrm{~m} / \mathrm{h}$ and $9.7 \mathrm{~h} /$ day, respectively. Generally, the area under consideration is characterized by a hot and dry summer with rare winter rainfall. 
Table 1. The climatological data of the study area (EL Kharga oasis meteorological station)

\begin{tabular}{|c|c|c|c|c|c|c|c|}
\hline \multirow[t]{2}{*}{ Month } & \multicolumn{2}{|c|}{ Temperature ${ }^{\circ} \mathrm{C}$} & \multirow{2}{*}{$\begin{array}{l}\text { Relative } \\
\text { Humidity }(\%)\end{array}$} & \multirow{2}{*}{$\begin{array}{l}\text { windspeed } \\
(\mathrm{n} / \mathrm{h})\end{array}$} & \multirow{2}{*}{$\begin{array}{l}\text { Sun shine } \\
\text { (h/day) }\end{array}$} & \multirow{2}{*}{$\begin{array}{l}\text { Rain fall } \\
(\mathrm{mm})\end{array}$} & \multirow{2}{*}{$\begin{array}{l}\text { Evaporation } \\
\text { (mm) }\end{array}$} \\
\hline & Highest & Lowest & & & & & \\
\hline January & 24.6 & 9.2 & 37 & 2.5 & 8.0 & 0.1 & 6.0 \\
\hline February & 27.7 & 10.6 & 27 & 2.7 & 8.5 & - & 5.4 \\
\hline March & 32.9 & 15 & 19 & 3.0 & 10.0 & - & 6.0 \\
\hline April & 35.9 & 18 & 17 & 3.1 & 10.4 & - & 5.4 \\
\hline May & 39.4 & 21.9 & 15 & 3.0 & 10.9 & - & 8.0 \\
\hline June & 42.4 & 24.5 & 14 & 2.4 & 12.6 & - & 10.4 \\
\hline July & 42.3 & 24.6 & 16 & 2.3 & 12.1 & - & 8.7 \\
\hline August & 44.1 & 25.3 & 17 & 2.7 & 10.1 & - & 9.1 \\
\hline September & 40.3 & 23.7 & 20 & 2.5 & 8.7 & - & 9.9 \\
\hline October & 34.5 & 19.4 & 23 & 2.6 & 8.4 & - & 9.9 \\
\hline November & 29.5 & 14.3 & 36 & 2.5 & 8.1 & - & 7.7 \\
\hline December & 24.3 & 9.7 & 38 & 2.7 & 8.0 & 0.1 & 6.6 \\
\hline Average & 34.8 & 18.0 & 23.3 & 2.7 & 9.7 & - & 7.76 \\
\hline Total & - & - & - & - & - & 0.20 & - \\
\hline
\end{tabular}

Egyption Meteorological Authority( 2014)

According to USDA (2014), the study area belongs to "Hyperthermic" temperature regime and "Torric "moisture regime.

The objective of this study is to identify the physiographic units morphological feutures and properties of soils south west Paris Oasis, Egypt with the aid of remote sensing technique.

\section{Materials and Methods}

\subsection{Study area:}

The area under consideration is located south west of Paris Oasis and west of Darb El-Arabaein main road between latitudes $24^{\circ} 20^{-}$and $24^{\circ} 40^{-} \mathrm{N}$ and longitudes $30^{\circ} 00^{-}$and $30^{\circ} 30^{-} \mathrm{E}$ Fig. (2) and occupies a total area of 178218 hectares.

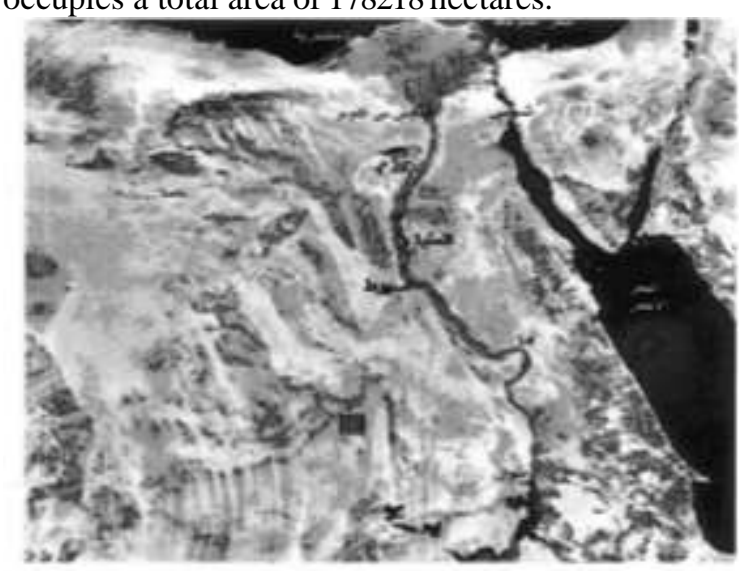

Fig 1 lecatioe map of the studied ara

\subsection{Physiographic analysis:}

The physiographic analysis using visual interpretation was carried with Landsat ETM of the year 2005, bands 4,3,2 as RGB.The visual analysis based

On developing techniques of Lueder (1959), Vink (1963),

Goosen(1967)and Sabins(1978).

\subsection{Field work:}

Twenty nine soil profiles were chosen to represent the main physiographic units of the studied area the studied profiles are illustrated in Fig. (2).

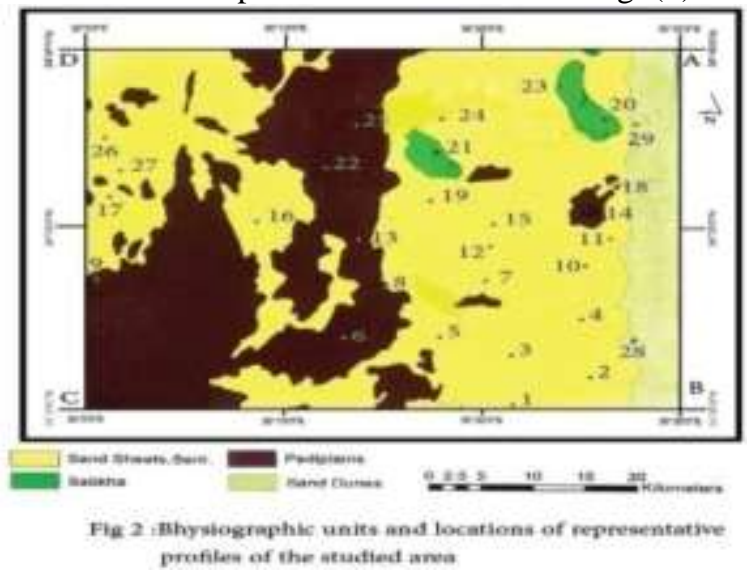

Soil profiles were dug to bedrock according to FAO (1994).

\subsection{Laboratory analysis:}

The soil samples were collected, air dried, crushed, sieved through a $2 \mathrm{~mm}$ sieve and subjected to physical and chemical analyses as follows:

Gravel contents were determined as percentage by volume.

Particle size distribution was carried out using sodium hexametaphosphate as adispersing agent according to Black (1965).

Soil reaction $(\mathrm{pH})$ wa $\mathrm{s}$ measured in the saturated soil paste (Richards, 1954).

Soil salinity (ECe) and soluble cations and anions were determined in saturated soil paste extract (Jackson, 1975) , except soluble sulphate anion which is calculated by subtracting total anions from total cations.

Organic matter contents were determined according to modified procedure in Jackson(1958). 
Gypsum contents were determined by precipitation with acetone according to Richards (1954).

Total carbonate contents were measured by Collin's Calcimeter according to Piper (1950).

Soil classification was conducted following up the USDA system (2014).

Land capability classification was done according to Sys and Verheye (1978)

Results and Discussion:

3.1-Physiograhic features:

The area under consideration has four landform units according to the visual interpretation of the Landsat satellite image namely, sand sheet, sabkha, pediplain and barchans dunes belts.

A- Sand sheet: Soils of the sand sheet are blanket deposits of sand (Robert and Julia, 1983) which are represented by soil profiles 1, 2, 3,4,5,7, $10,11,12,15,16,17,18,19,24,26$ and 27 . It covers about 95042 hectares representing $53.33 \%$ of the total studied area. The main surface features are fine gravel and very few rock outcrops, while thin loose sand sheet cover the unit.

The slope gradient differed between nearly level and sloping.

Soil color through the different layers of the studied profiles ranged between light gray and yellow (Tabe2).

The very pale brown color is the predominant. Soil depth varied from deep to shallow with textural class varied from sand to clay loam and gravel contents raging between 5.0 and $40 \%$ with few pedognic features of crystalline gypsum in some profiles .

Organic matter content varied from 0.05 to $0.34 \%$ (Table3), which represent trace constituents and reflects the arid and thermic conditions. Contents of total carbonate ranged between 0.4and 8.2\%. Gypsum contents range from0.06to5.85\%, forming a Gypsic horizon in soil profiles3and 12 (Table 3 ).

Soil chemical characteristics, in Table (4) show that the soil reaction $(\mathrm{pH})$ values ranged from 7.04 to 8.33 .

Soil Salinity (ECe) values varied widely between 3.51 and $75.1 \mathrm{dS} / \mathrm{m}$ at $25 \mathrm{C}^{0}$ of soil paste extract .Generally, soluble contents of anions appeared the following trend $\mathrm{Cl}^{-}>$SÖ4 $>\mathrm{HCŌ} 3$ while soluble cations followed the sequence. $\mathrm{Na}^{+}>\mathrm{Ca}^{2+}>$ $\mathrm{Mg}^{2+}>\mathrm{K}^{+}$.According to USDA, (2014),

soil profiles of this unit are classified as: Coarse loamy, mixed, hyerthermic, TypicTorriorthents in profiles 1,11and 16 ;Fine loamy, mixed, hyperthermic, TypicTorriortherents in profiles4, 15and26; Sandy, , mixed,hyperthermic, in profiles 10, 17,18, and 27 ;Sandy, over loamy , mixed, hyperthermic, TypicTorriorthents i n profiles 2 and 24 ; Fine loamy, mixed, hyperthermic, Lithic Torriorthentsin profiles5,7and19; Sandy, mixed, hyperthermic, Lithic Haplogypsids in profiles 3 and 12.

\section{B-Sabkha:}

According to Robert and Julia (1983) sabkha in the rock records faces may be indicated by evaporates, absence of fossils, thin flat-pebble conglomerates, stromatolitic laminae, mud cracks, and dolomitization.

Profiles 20, 21 and 23 are the representative profiles of Sabkha unit It covers about 3701 hectare representing $2.07 \%$ of the total studied area.

Data in Fig (2) appear locations of the studied profiles, while, data in Table (2) show the morphological features.

Soils of profile 20 have a thin layer of slightly hard of salt crust, while the others have medium and coarse gravel.

The slope gradient is nearly level. Soil color ranged from10YR7/2to10YR7/8.

Textural classes varied widely from loamy sand to clay. There are some pedoginic features such as crystalline salts with or without gypsum crystals. The Soil depth tended to be moderately deep.

The physical properties illustrated in Table (3) show that organic matter content varied from 0.05 to $0.28 \%$, total carbonate content ranged between 1.5 and $4.5 \%$, gravel contents ranged from 2 to $15 \%$, while gypsum contents differed from 0.89 and $8.54 \%$.

Results revealed the presence of a gypsic horizon through both profiles 20 and 23 .

Chemical properties (Table4) illustrated that $\mathrm{pH}$ values ranged between 7.11 and8.04. Soil salinity (EC) varied widely between 3.1 and $189 \mathrm{dS} / \mathrm{m}$.The distribution of soluble anions followed either the ascending order $\mathrm{SO}_{4}^{2-}>\mathrm{Cl}^{-}>\mathrm{HCO}_{3}^{-}$or $\mathrm{Cl}^{-}>$ $\mathrm{SO}_{4}^{2-}>\mathrm{HCO}_{3}^{-}$, while the distribution of soluble cations followed the ascending order $\mathrm{Na}^{+}>$ $\mathrm{Ca}^{2+}>\mathrm{Mg}^{2+}<\mathrm{K}^{+}$.

Soils of all representative profiles of the unit have a salic horizon. According to USDA,(2014), soils of Sabkha unit are classified to the family levels as follows: Fine clayey, mixed, hyperthermic, Gypsic Haplosalids, in profiles 20 and 23, a n d sandy, mixed, hyperthermic, Typic Haplosalids, in profil21

\section{C-Pediplain.}

Pediplain is an extensive thinly alleviated erosion surface formed in a desert region by coalescence of two or more adjacent pediments and occasional desert domes and representing the end result of the mature stage of the arid region cycle, according to Robert and Julia (1983).

The total area of the unit under consideration is about 63468 hectares which represents( $35.60 \%$ )of total area .Soils profiles $6,8,9,13,14,22$, and 25 are the representative profiles of pediplain unit. There are few rock outcrops on the surface and slope gradient ranging from gently sloping to sloping (Table2). Soil color varied between10YR7/3.and 10YR7/8. The pedoginic features of few soft lime or crystalline gypsum. Are observed Soils texture classes (Table3) differed from sand to sandy clay 
loam and soil depth varied from shallow to moderately deep. Organic matter content ranged from 0.08 to $0.27 \%$. Gypsum content varied from0. 13 to $3.60 \%$, while lime content ranged between 1.6 and $6.4 \%$, on the other hand gravel contents differed from 2.0to50.0\%. Soil reaction $(\mathrm{pH})$ ranged between 7.08 and 8.23 (Table4) .EC values varied widely from 2.9 to $101.6 \mathrm{dS} / \mathrm{m}$. Generally, $\mathrm{t} \mathrm{h} \mathrm{e}$ distribution of soluble anions was: $\mathrm{CL}^{-}>\mathrm{SO}_{4}^{2-}>$ $\mathrm{HCO}_{3}^{-}$, and $\mathrm{Na}+>\mathrm{Ca}^{2}+>\mathrm{Mg}^{2}+>\mathrm{K}+$ for the soluble cations . According to USDA (2014) the soils of this unit are classified as: Sandy, mixed, hyperthermic, TypicTorriothents, in profiles 6,8 and 25 , Fine loamy,mixed, hyperthermic. Lithic Torriothents, in profiles 9 and 22, coarse loamy, mixed, hyperthermic. Lithic Torriothents, in profile 13and course loamy, mixed hyperthermic. TypicTorriothents, in profile 14;

\section{D-Barchan dunes belts.}

Dunes is a sand waves formed on a stream bed, traverse to the direction of flow and traveling downstream of sand from the genetic upstream slope and deposition on the steep downstream slope, (Robert and Julia,1983). This unit covers about 16047 hectares, which represents $9.00 \%$ of total area . This unit was represented by profiles 28 and 29. The Soils have steep sloping, yellow color, sand texture class, singly grains structure, and have not any diagnostic horizons(Table2).The gravel contents (Table3) varied between 1.0 and $2.0 \%$, gypsum contents from 0.08 to $0.09 \%$, calcium carbonate from 0.13 to $0.16 \%$, while organic matter from 0.03 to $0.04 \%$.

Soil reaction $(\mathrm{pH})$ differed from 8.1 to 8.2 (Table 4) . EC ranged between 1.4 and $1.7 \mathrm{dS} / \mathrm{m}$ soluble anions followed the sequence of $\mathrm{CL}^{-}>\mathrm{SO}_{4}^{2-}>\mathrm{HCO}_{3}^{-}$ while souble cation follows: $\mathrm{Na}+>\mathrm{Ca}^{2}+>\mathrm{Mg}^{2}+>\mathrm{K}+$. According USDA (2014), the soils of this unit are classified as: siliceous, hyperthermic, Lithic Torripsamments,

\section{3-2-Land evaluation}

According to the parametric of Sys and Verheye (1978) the study soils are evaluated (Table, 5) as follows:

Soils of sand sheet have current suitability varied of not suitable (profile 3 and 5), marginal suitable (profiles 2,4,7,10,11,12,16,18, 19 and 24) and moderately suitable (profiles1, 15, 17, 26 and 27) .The abundance limitations are soil depth, texture and salinity and alkalinity. The potential suitability would be highly suitable ( S1) for profile 17, 24 and 26; moderately suitable(S2) for profiles $1,10,11,15,16$ and 27 and marginally suitable for profiles 2, 3, 4, 5,7, 12, 18 and 19. The current suitability for the pediplain unit is marginally suitability (S3) except profile 25 which not suitable (N1). That the soils of this unit Potential would be moderately suitable (S2) except profiles 6, 9, 22 and 25 which would be marginally suitable (S3).

Soils of sabkha are marginally (S3) and moderately (S2) suitable in their current and potential conditions respectively.

Soils of sand dunes appear not suitable (N2) in both current and potential suitability. 
Table 2. Morphological description of the studied soil profiles

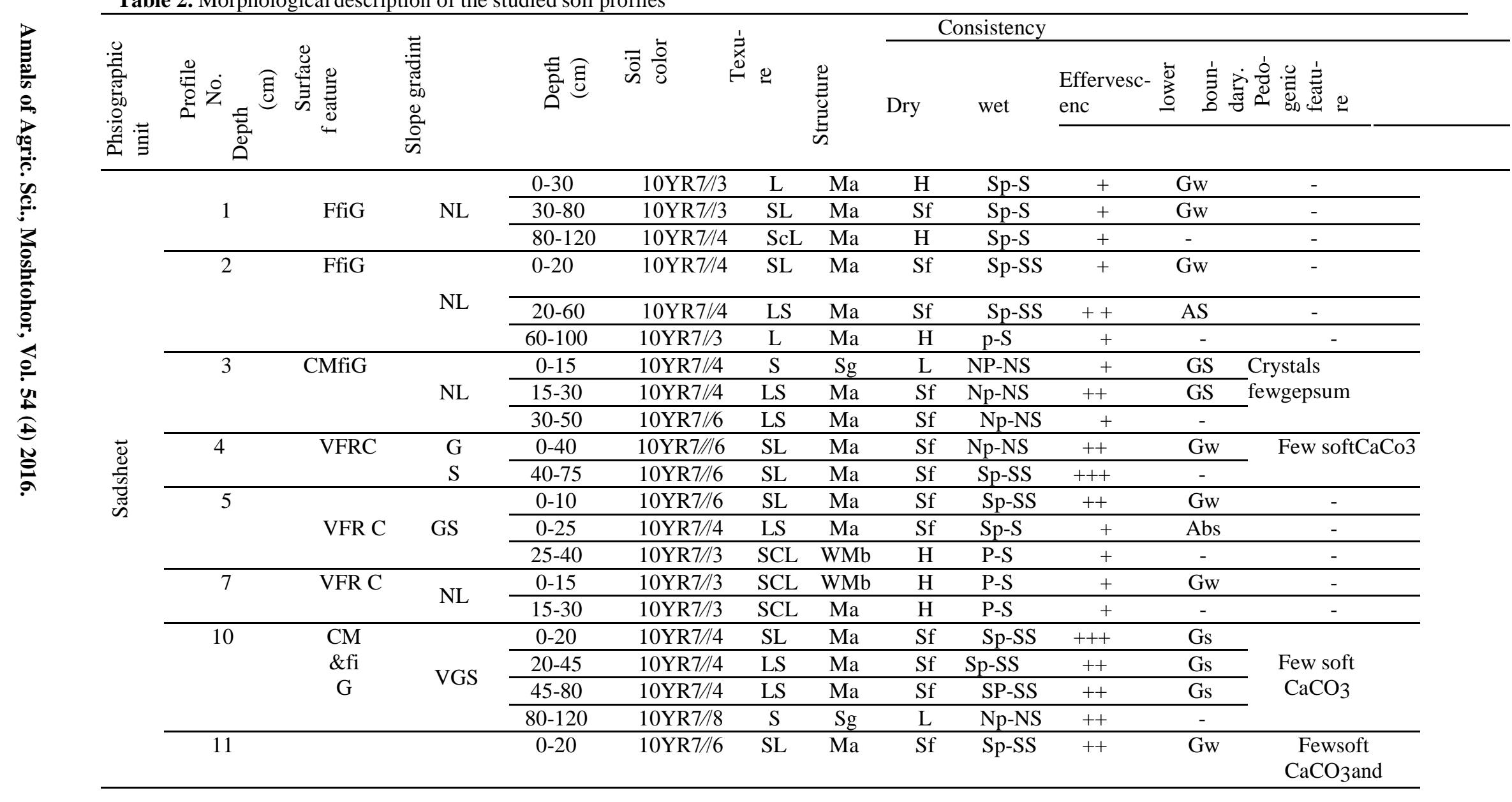


Table 2. Cont

\begin{tabular}{|c|c|c|c|c|c|c|c|c|c|c|c|c|}
\hline Physi- & Pro- & Surfa- & Slope & Depth & Soil Color & Tex- & Stru- & Consis & & Effer- & Lower & Pedogenic \\
\hline $\begin{array}{c}\text { Ograp } \\
\text { hic unit }\end{array}$ & $\begin{array}{l}\text { file } \\
\text { No. }\end{array}$ & $\begin{array}{c}\text { ceFeatu- } \\
\text { re }\end{array}$ & Gradient & $(\mathrm{cm})$ & & ture & cture & Dry & Wet & $\begin{array}{l}\text { vesc- } \\
\text { ence }\end{array}$ & boundary & Feature \\
\hline \multirow{26}{*}{ 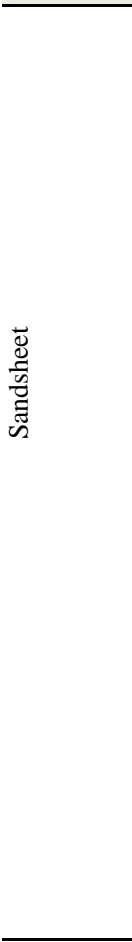 } & & CM\&fiG & NL & $20-45$ & 10YR7//4 & SL & $\mathrm{Ma}$ & $\mathrm{Sf}$ & Sp-SS & ++ & Gs & Few gypsum crystals \\
\hline & & & & $45-70$ & 10YR7//6 & SL & Ma & $\mathrm{Sf}$ & Sp-SS & ++ & Gw & \\
\hline & & & & $70-90$ & $10 \mathrm{YR} 7 / 3$ & SCL & $\mathrm{Ma}$ & $\mathrm{H}$ & $\mathrm{p}-\mathrm{S}$ & ++ & - & \\
\hline & 12 & CM\&fi G & NL & $0-15$ & $10 \mathrm{YR} 7 / / 3$ & SCL & $\mathrm{WMb}$ & $\mathrm{H}$ & $\mathrm{P}-\mathrm{S}$ & +++ & Gw & Few $\mathrm{CaCO}_{3}$ Nodules and few \\
\hline & & & & $15-30$ & $10 \mathrm{YR} 7 / / 8$ & $\mathrm{~S}$ & $\mathrm{Sg}$ & $\bar{L}$ & Np-NS & ++ & - & gypsum crystals \\
\hline & 15 & VFfiG & VGS & $0-30$ & $10 \mathrm{yr} 7 / 3$ & SCL & $\mathrm{Ma}$ & $\mathrm{H}$ & $\mathrm{p}-\mathrm{S}$ & + & $\mathrm{Gw}$ & - \\
\hline & & & & $30-70$ & $10 \mathrm{YR} 7 / / 6$ & SL & $\mathrm{Ma}$ & $\mathrm{Sh}$ & Sp-SS & ++ & Gw & \\
\hline & & & & $70-110$ & $10 \mathrm{YR} 7 / / 4$ & LS & $\mathrm{Ma}$ & Sf & Sp-SS & + & - & \\
\hline & 16 & FfiG & GS & $0-30$ & $10 \mathrm{YR} 7 / / 4$ & LS & $\mathrm{Ma}$ & $\mathrm{Sf}$ & Sp-SS & ++ & GS & - \\
\hline & & & & $30-50$ & 10YR7//6 & SL & $\mathrm{Ma}$ & Sh & Sp-SS & + & Gw & - \\
\hline & & & & $50-70$ & $10 \mathrm{YR} 7 / / 6$ & SL & $\mathrm{Ma}$ & $\mathrm{Sh}$ & Sp-SS & + & - & - \\
\hline & 17 & $\overline{\mathrm{FfiG}}$ & GS & $0-20$ & $10 \mathrm{YR} 7 / / 4$ & SCL & $\mathrm{Ma}$ & $\mathrm{H}$ & $\mathrm{P}-\mathrm{S}$ & +++ & $\mathrm{Cw}$ & Few soft $\mathrm{CaCO} 3$ and few gypsum crystals \\
\hline & & & & $20-60$ & $10 \mathrm{YR} 7 / / 6$ & LS & $\mathrm{Ma}$ & Sf & Sp-SS & + & GS & \\
\hline & & & & $60-90$ & 10YR7//6 & SL & $\mathrm{Ma}$ & Sf & Sp-SS & + & - & \\
\hline & 18 & $\overline{\mathrm{FfiG}}$ & $\mathrm{S}$ & $0-20$ & $10 \mathrm{YR} 7 / / 6$ & SL & $\mathrm{Ma}$ & Sf & Sp-SS & + & $\mathrm{Cw}$ & \\
\hline & & & & $20-60$ & $10 \mathrm{YR} 7 / / 4$ & LS & $\mathrm{Ma}$ & Sf & Np-NS & + & - & \\
\hline & 19 & FfiG & GS & $0-20$ & $10 \mathrm{YR} 7 / / 6$ & SL & $\mathrm{Ma}$ & Sh & Sp-SS & ++ & GW & Fewgypsum \\
\hline & & & & $20-40$ & $10 \mathrm{YR} 7 / / 6$ & SL & $\mathrm{Ma}$ & Sh & SP- SS & ++ & - & crystals \\
\hline & 24 & FRC & NL & $0-30$ & 10YR7//8 & $\mathrm{S}$ & $\mathrm{Sg}$ & $\mathrm{L}$ & Np-NS & ++ & AS & \\
\hline & & & & $30-80$ & $10 \mathrm{YR} 7 / / 3$ & $\mathrm{CL}$ & $\mathrm{WMb}$ & $\mathrm{H}$ & $\mathrm{p}-\mathrm{S}$ & + & Gw & \\
\hline & & & & $80-100$ & 10YR7//4 & CL & $\mathrm{WMb}$ & $\mathrm{H}$ & $\mathrm{p}-\mathrm{S}$ & + & - & \\
\hline & 26 & FRC & $\mathrm{S}$ & $0-20$ & $10 \mathrm{yR} 7 / 3$ & SCL & Ma & $\mathrm{H}$ & $\mathrm{Sp}-\mathrm{S}$ & ++ & GW & Few soft \\
\hline & & & & $20-80$ & $10 \mathrm{YR} 7 / 3$ & $\mathrm{~L}$ & Ma & $\mathrm{H}$ & Sp-S & ++ & - & $\mathrm{CaCO}_{3}$ \\
\hline & 27 & FRC & GS & $0-30$ & 10YR7//4 & LS & $\mathrm{Ma}$ & Sf & Sp-SS & + & GS & - \\
\hline & & & & $30-80$ & 10YR7//6 & $\mathrm{S}$ & $\mathrm{Sg}$ & $\mathrm{L}$ & NPNS & + & Gw & - \\
\hline & & & & $80-130$ & 10YR7//6 & $\mathrm{S}$ & $\mathrm{Sg}$ & $\mathrm{L}$ & Np-NS & + & - & - \\
\hline $\begin{array}{l}\text { Sabk- } \\
\text { ha }\end{array}$ & 20 & $\mathrm{SC}$ & $\mathrm{NL}$ & $0-20$ & 10YR7/3 & $\mathrm{CL}$ & WMb & $\mathrm{H}$ & P-S & + & $\mathrm{CW}$ & $\begin{array}{c}\text { Few } \\
\text { Gypsum }\end{array}$ \\
\hline
\end{tabular}


Table 2 Cont.

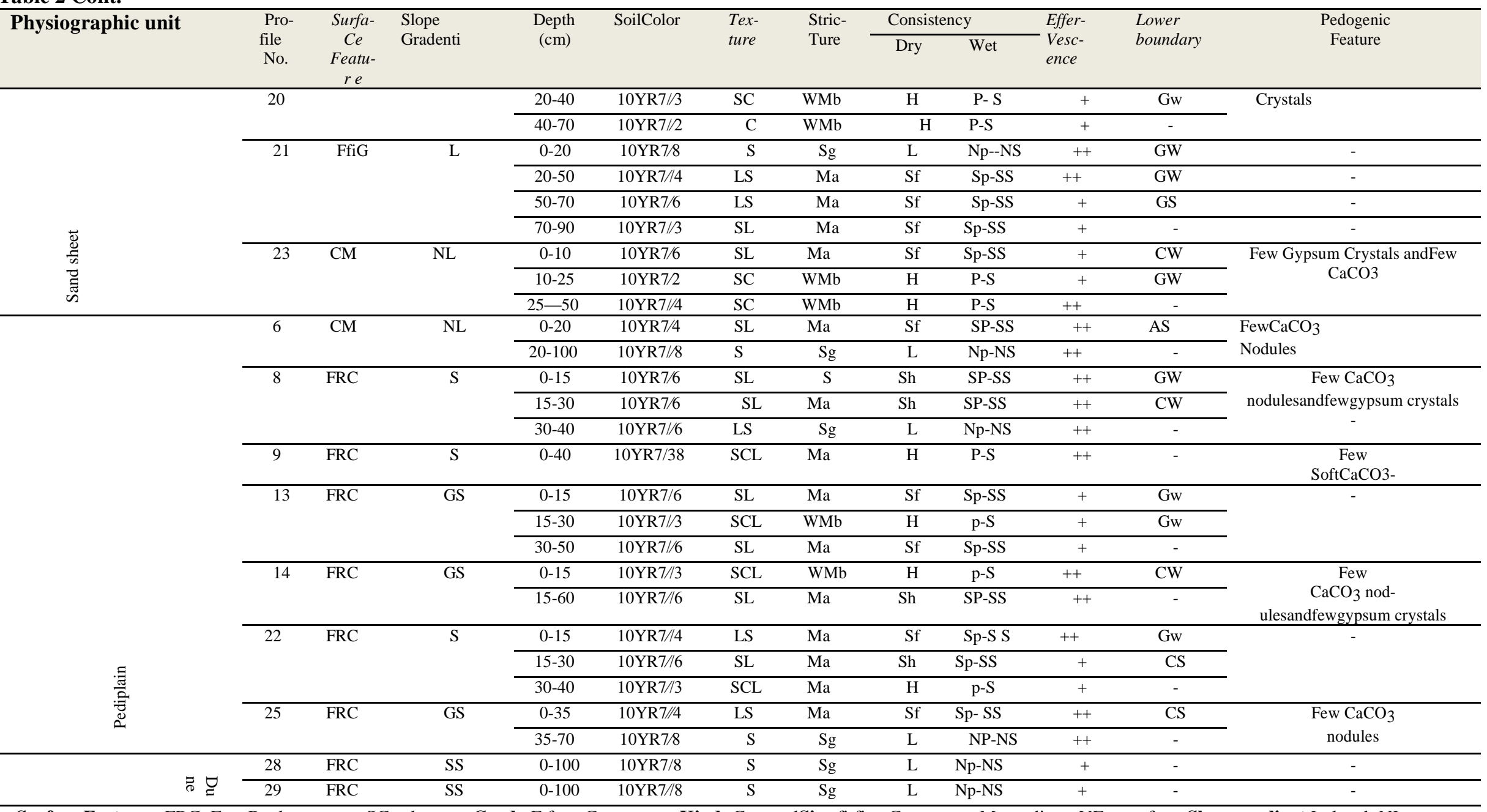

nearlylevel, S: sloping, GS:gentlysloping, SS: stronglysloping, VGS:verygentlysloping -Texture:L: loam, SCL: sandyclayloam, LS: loamysand, SL: sandyloam, CL: clayloam, S: sand,C: clay, SC: sandyclay -Structure: Ma.Massive; Sg: Singlegrain;WMb; Weak medium blocky -Consistence:Dry; Sf. Soft. H:.hard. .L:.loose, Sh. Slightlyhard.Vh. Veryhard Wet.Plasticity

.P,Plastic,Sp.SlightlyplasticNp: Nonplastic.VP,Very plastic.Stickness,S,sticky.SS.slightly.sticky.NS..non.sticky.VS, verysticky Effervescence:+slight++.moderate+++strong-..Boundary: As. Abrupt Smooth .GW: Gradual Wavy.GS :Gradual smooth. AW: Abrupt Wavy .CW :ClearWavy. 
Table 3. Some physical properties of the studied area

\begin{tabular}{|c|c|c|c|c|c|c|c|c|c|c|c|}
\hline \multirow{2}{*}{ 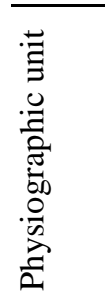 } & \multirow{2}{*}{ 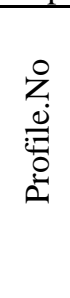 } & \multirow[b]{2}{*}{ 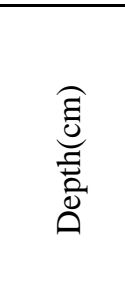 } & \multicolumn{4}{|c|}{ Particlesizedistribution(\%) } & \multirow[b]{2}{*}{ 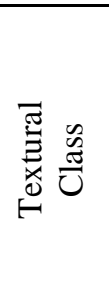 } & \multirow[b]{2}{*}{ 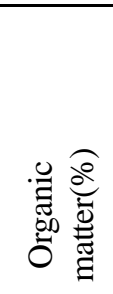 } & \multirow[b]{2}{*}{ 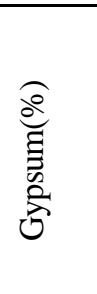 } & \multirow[b]{2}{*}{$\frac{\widehat{e}}{\tilde{e}}$} & \multirow[b]{2}{*}{$\frac{\widehat{0}}{\stackrel{0}{0}}$} \\
\hline & & & 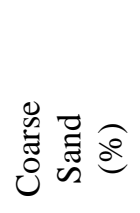 & 局蛋 & $\stackrel{\approx}{a}$ & 胥 $\widehat{\varrho}$ & & & & & \\
\hline \multirow{20}{*}{ 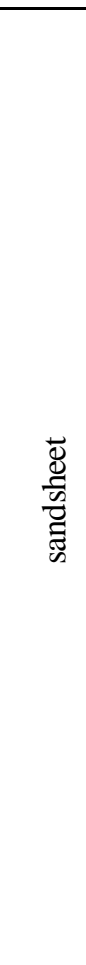 } & \multirow[b]{3}{*}{1} & $0-30$ & 28.76 & 16.98 & 34.02 & 20.24 & $\mathrm{~L}$ & 0.16 & 0.44 & 0.40 & 5.00 \\
\hline & & $30-80$ & 23.35 & 21.65 & 39.42 & 15.58 & SL & 0.22 & 0.30 & 3.50 & 5.00 \\
\hline & & $80-120$ & 38.16 & 21.11 & 16.44 & 24.29 & SC L & 0.07 & 0.22 & 2.40 & 20.00 \\
\hline & \multirow[t]{3}{*}{2} & $0-20$ & 42.55 & 37.70 & 6.72 & 13.03 & SL & 0.07 & 0.86 & 4.7 & 30.00 \\
\hline & & $20-60$ & 50.68 & 36.03 & 7.09 & 6.20 & LS & 0.09 & 0.71 & 2.5 & 10.00 \\
\hline & & $60-100$ & 12.36 & 26.50 & 33.54 & 27.60 & $\mathrm{~L}$ & 0.24 & 0.29 & 3.2 & 10.00 \\
\hline & \multirow[t]{3}{*}{3} & $0-15$ & 34.69 & 54.14 & 6.57 & 4.60 & $S$ & 0.12 & 5.85 & 3.50 & 5.00 \\
\hline & & $15-30$ & 37.80 & 42.96 & 5.38 & 13.86 & LS & 0.25 & 5.40 & 4.00 & 5.00 \\
\hline & & $30-50$ & 30.50 & 48.39 & 13.92 & 7.19 & LS & 0.18 & 2.91 & 1.80 & 10.00 \\
\hline & \multirow[t]{2}{*}{4} & $0-40$ & 36.42 & 34.35 & 13.87 & 15.36 & SL & 0.06 & 0.97 & 6.50 & 10.00 \\
\hline & & $40-75$ & 30.83 & 30.83 & 7.32 & 18.08 & SL & 0.15 & 0.37 & 8.20 & 15.00 \\
\hline & \multirow[b]{3}{*}{5} & $0-10$ & 33.73 & 23.16 & 25.63 & 17.48 & SL & 0.09 & 0.86 & 4.00 & 5.00 \\
\hline & & $10-20$ & 52.66 & 25.38 & 9.51 & 12.93 & LS & 0.13 & 0.49 & 3.50 & 10.00 \\
\hline & & $20-40$ & 43.60 & 27.19 & 8.54 & 20.67 & SCL & 0.17 & 0.55 & 1.80 & 20.00 \\
\hline & \multirow[b]{2}{*}{7} & $0-15$ & 20.26 & 28.35 & 19.40 & 31.99 & SCL & 0.25 & 0.34 & 1.80 & 10.00 \\
\hline & & $15-30$ & 18.02 & 39.69 & 22.03 & 20.26 & SCL & 0.20 & 0.42 & 2.00 & 10.00 \\
\hline & \multirow{4}{*}{10} & $0-20$ & 32.03 & 40.92 & 10.38 & 16.67 & SL & 0.14 & 1.94 & 8.00 & 2.00 \\
\hline & & $20-45$ & 30.93 & 51.65 & 7.59 & 9.83 & LS & 0.17 & 1.66 & 6.20 & 2.00 \\
\hline & & $45-80$ & 46.53 & 34.96 & 6.84 & 11.67 & LS & 0.12 & 1.40 & 4.80 & 10.00 \\
\hline & & $80-120$ & 49.82 & 38.45 & 4.56 & 7.17 & $\mathrm{~S}$ & 0.07 & 0.14 & 4.00 & 15.00 \\
\hline
\end{tabular}


Table(3)Cont

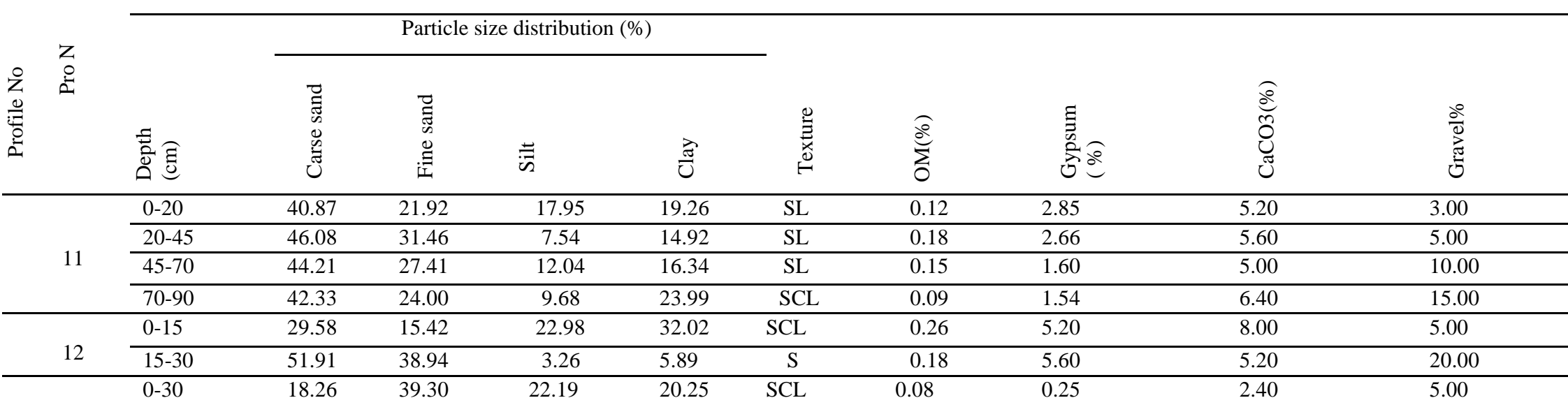

\begin{tabular}{|c|c|c|c|c|c|c|c|c|c|c|c|}
\hline \multirow{6}{*}{\multicolumn{2}{|c|}{$\frac{\mathbb{d}}{n}$}} & $30-70$ & 15.99 & 43.36 & 21.91 & 18.74 & SL & 0.25 & 0.29 & 4.5 & 20.00 \\
\hline & & $70-110$ & 40.35 & 42.84 & 4.06 & 12.75 & LS & 0.16 & 0.82 & 1.60 & 40.00 \\
\hline & & $0-30$ & 24.14 & 56.04 & 6.88 & 12.941 & LS & 0.08 & 0.06 & 4.80 & 2.00 \\
\hline & & $30-50$ & 15.23 & 36.05 & 32.41 & 15.77 & SL & 0.16 & 1.63 & 3.00 & 5.00 \\
\hline & & $50-70$ & 37.84 & 30.86 & 22.74 & 8.56 & SL & 0.11 & 1.37 & 2.40 & 7.00 \\
\hline & & $0-20$ & 40.15 & 27.48 & 9.81 & 22.56 & SCL & 0.09 & 1.57 & 8.00 & 5.00 \\
\hline స్ & \multirow[t]{2}{*}{17} & $20-60$ & 56.45 & 26.08 & 4.53 & 12.94 & LS & 0.24 & 3.95 & 2.40 & 20.00 \\
\hline & & $60-90$ & 54.68 & 25.28 & 5.66 & 14.38 & SL & 0.07 & 2.70 & 1.60 & 20.00 \\
\hline & \multirow[b]{2}{*}{18} & $0-20$ & 45.37 & 32.55 & 11.57 & 10.51 & SL & 0.06 & 2.28 & 3.20 & 30.00 \\
\hline & & $20-60$ & 47.12 & 29.33 & 10.79 & 12.67 & LS & 0.14 & 1.31 & 2.80 & 10.00 \\
\hline & \multirow[b]{2}{*}{19} & $0-20$ & 53.67 & 25.47 & 4.26 & 16.60 & SL & 0.24 & 2.80 & 4.00 & 15.00 \\
\hline & & $20-40$ & 42.57 & 32.65 & 6.30 & 18.48 & SL & 0.16 & 0.26 & 3.40 & 10.00 \\
\hline & \multirow{4}{*}{24} & $0-30$ & 48.72 & 44.16 & 3.65 & 3.47 & $\mathrm{~S}$ & 0.17 & 2.80 & 4.00 & 25.00 \\
\hline & & $30-80$ & 9.97 & 27.88 & 31.19 & 30.98 & CL & 0.34 & 2.40 & 2.40 & 5.00 \\
\hline & & $80-100$ & 7.06 & 21.75 & 38.35 & 32.84 & CL & 0.22 & 1.72 & 1.80 & 5.00 \\
\hline & & $0-20$ & 42.27 & 29.70 & 7.00 & 21.03 & SCL & 0.09 & 0.75 & 6.20 & 5.00 \\
\hline & 26 & $20-80$ & 12.11 & 27.00 & 35.96 & 24.93 & $\mathrm{~L}$ & 0.24 & 1.52 & 4.00 & 10.00 \\
\hline
\end{tabular}




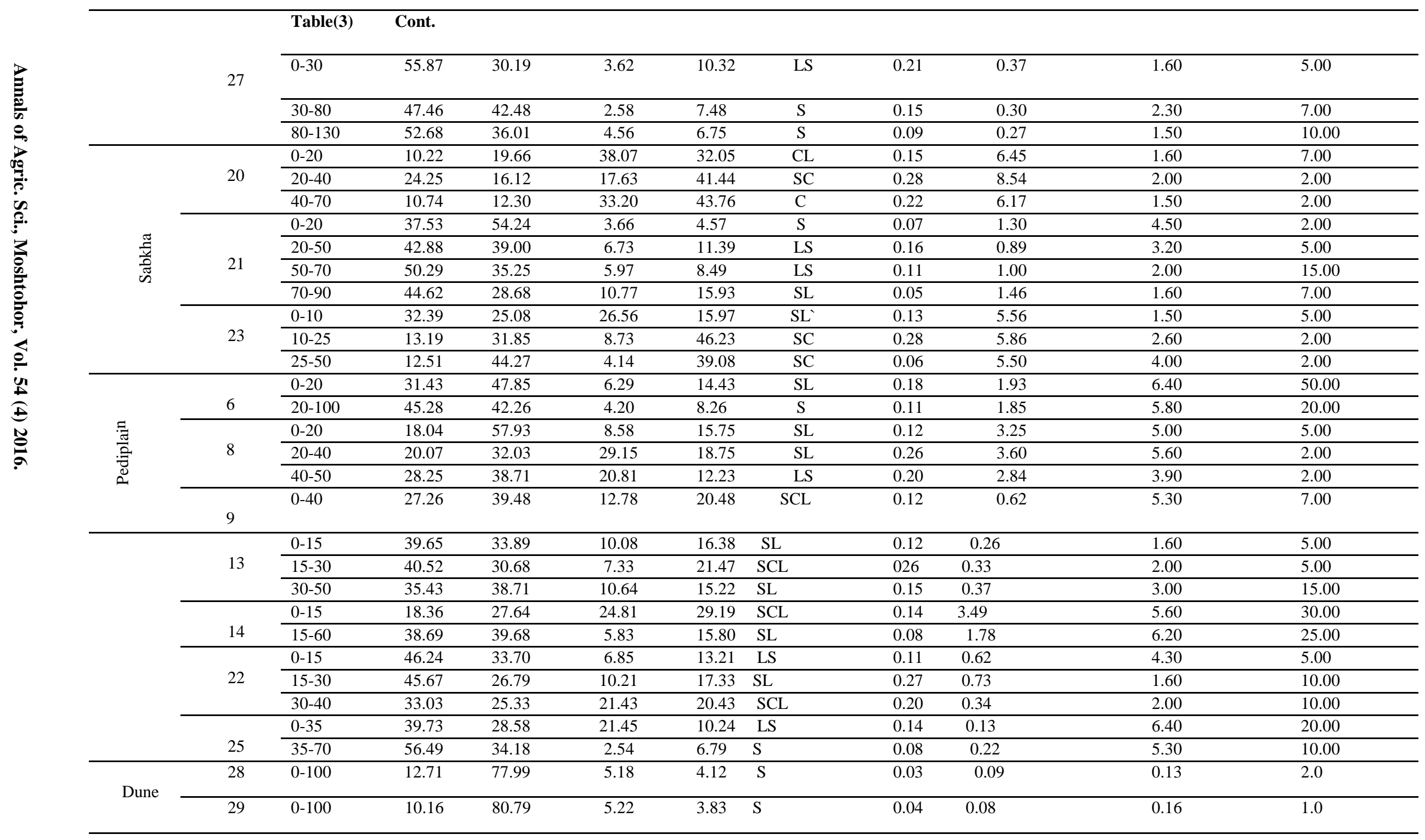


Table 4. Chemical properties of the studied area

\begin{tabular}{|c|c|c|c|c|c|c|c|c|c|c|c|c|c|c|}
\hline \multirow{2}{*}{$\begin{array}{l}\text { Physiographic } \\
\text { unit }\end{array}$} & \multirow{2}{*}{$\begin{array}{l}\text { Profile } \\
\text { No. }\end{array}$} & \multirow{2}{*}{$\begin{array}{c}\text { Depth } \\
\text { (cm) }\end{array}$} & \multirow{2}{*}{ PH } & \multirow{2}{*}{$\begin{array}{c}\mathrm{EC} \\
(\mathrm{dS} / \mathrm{m})\end{array}$} & \multicolumn{4}{|c|}{ Soluble Anions (me/l) } & \multicolumn{4}{|c|}{ Soluble Cations(me/l) } & \multirow[b]{2}{*}{ SAR } & \multirow[b]{2}{*}{ ESP } \\
\hline & & & & & $\mathrm{CO} 3=$ & $\mathrm{HCO}_{3}^{-}$ & $\mathrm{Cl}^{-}$ & $\mathrm{SO}_{4}^{-2}$ & $\mathrm{Ca}^{+2}$ & $\mathrm{Mg}^{+2}$ & $\mathrm{Na}^{+}$ & $\mathrm{K}^{+}$ & & \\
\hline \multirow{28}{*}{ 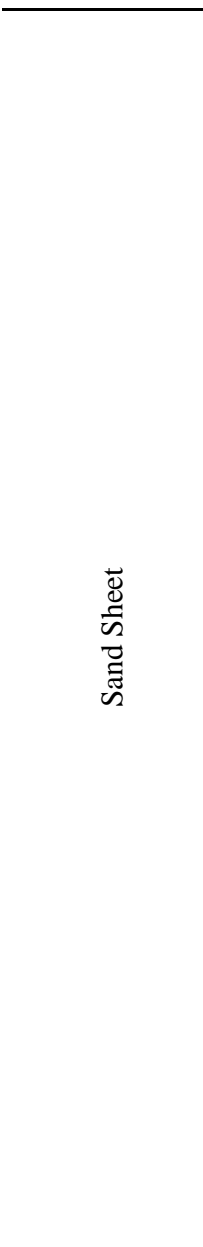 } & \multirow{3}{*}{1} & $0-30$ & 8.08 & 5.15 & - & 3.00 & 21.00 & 30.71 & 26.60 & 7.70 & 20.00 & 0.40 & 4.83 & 5.53 \\
\hline & & $30-80$ & 8.19 & 14.70 & - & 2.00 & 118.00 & 59.80 & 42.60 & 14.60 & 121.00 & 1.60 & 22.66 & 24.34 \\
\hline & & $80-120$ & 8.06 & 16.10 & - & 1.50 & 102.50 & 73.90 & 59.20 & 15.10 & 110.00 & 1.60 & 18.06 & 20.24 \\
\hline & \multirow{3}{*}{2} & $0-20$ & 7.99 & 14.61 & - & 3.00 & 116.00 & 56.20 & 65.60 & 16.70 & 91.60 & 1.30 & 14.29 & 16.54 \\
\hline & & $20-60$ & 8.15 & 14.36 & - & 2.00 & 114.00 & 59.20 & 57.90 & 20.10 & 96.10 & 1.10 & 15.50 & 17.76 \\
\hline & & $60-100$ & 8.07 & 8.81 & & 1.00 & 56.00 & 39.60 & 26.70 & 11.10 & 58.00 & 0.80 & 13.33 & 15.54 \\
\hline & \multirow{3}{*}{3} & $0-15$ & 8.11 & 27.20 & - & 3.50 & 180.00 & 142.90 & 134.00 & 56.30 & 134.00 & 2.10 & 13.74 & 15.97 \\
\hline & & $15-30$ & 7.41 & 4.90 & - & 2.00 & 21.00 & 27.95 & 22.60 & 8.00 & 20.00 & 0.35 & 5.12 & 5.92 \\
\hline & & $30-50$ & 7.32 & $42-60$ & - & 1.00 & 270.00 & 240.20 & 216.50 & 56.50 & 234.00 & 3.90 & 20.06 & 22.07 \\
\hline & \multirow[t]{2}{*}{4} & $0-40$ & 8.33 & 11.91 & - & 3.00 & 80.00 & 69.60 & 51.30 & 19.20 & 81.00 & 1.10 & 13.63 & 15.86 \\
\hline & & $40-75$ & 8.10 & 7.46 & - & 2.50 & 45.00 & 34.10 & 25.80 & 10.20 & 45.00 & 0.60 & 10.61 & 12.78 \\
\hline & \multirow{3}{*}{5} & $0-10$ & 7.91 & 34.00 & - & 3.50 & 235.20 & 203.30 & 194.80 & 29.10 & 215.00 & 3.10 & 20.32 & 23.06 \\
\hline & & $10-25$ & 8.11 & 37.5 & - & 2.50 & 241.00 & 237.50 & 200.50 & 86.10 & 194.15 & 0.25 & 16.22 & 19.31 \\
\hline & & $25-40$ & 7.54 & 40.90 & - & 1.50 & 220.80 & 310.40 & 246.00 & 115.20 & 167.30 & 4.20 & 12.45 & 15.51 \\
\hline & \multirow[t]{2}{*}{7} & $0-15$ & 7.63 & 29.50 & - & 2.50 & 225.00 & 123.40 & 88.10 & 29.50 & 230.00 & 2.30 & 29.98 & 30.05 \\
\hline & & $15-30$ & 7.54 & 7.95 & - & 1.00 & 52.00 & 46.45 & 38.20 & 10.60 & 50.00 & 0.65 & 10.12 & 12.02 \\
\hline & \multirow{4}{*}{10} & $0-20$ & 7.97 & 55.00 & - & 3.50 & 559.00 & 97.50 & 46.20 & 780.00 & 540.90 & 4.90 & 67.37 & 49.52 \\
\hline & & $20-45$ & 8.13 & 24.58 & - & 3.00 & 27.075 & 38.20 & 26.61 & 37.70 & 245.30 & 2.30 & 43.27 & 38.49 \\
\hline & & $45-80$ & 7.70 & 18.70 & - & 2.00 & 175.00 & 55.05 & 40.30 & 10.10 & 180.00 & 1.60 & 35.85 & 34.05 \\
\hline & & $80-120$ & 7.60 & 18.30 & & 1.00 & 120.00 & 102.80 & 72.10 & 25.30 & 125.00 & 1.40 & 17.90 & 20.09 \\
\hline & \multirow{4}{*}{11} & $0-20$ & 7.48 & 28.90 & - & 3.00 & 240.00 & 93.80 & 69.00 & 35.90 & 230.00 & 1.90 & 20.77 & 22.71 \\
\hline & & $20-45$ & 7.53 & 32.80 & - & 2.50 & 290.60 & 100.50 & 78.40 & 40.50 & 272.40 & 2.30 & 21.77 & 23.58 \\
\hline & & $45-70$ & 7.47 & 63.30 & - & 2.00 & 531.60 & 230.00 & 200.60 & 79.00 & 480.00 & 5.40 & 40.61 & 36.96 \\
\hline & & $70-90$ & 7.35 & 25.80 & - & 1.00 & 192.70 & 115.90 & 85.00 & 54.90 & 167.60 & 1.80 & 2.04 & 22.06 \\
\hline & \multirow[t]{2}{*}{12} & $0-15$ & 8.04 & 7.00 & - & 3.50 & 16.50 & 56.55 & 46.10 & 9.80 & 20.00 & 0.65 & 3.78 & 5.28 \\
\hline & & $15-30$ & 7.62 & 5.60 & - & 2.00 & 19.00 & 38.80 & 31.80 & 6.42 & 21.00 & 0.58 & 4.81 & 6.62 \\
\hline & \multirow[t]{2}{*}{15} & $0-30$ & 8.18 & 9.7 & - & 3.00 & 185.00 & 138.40 & 119.10 & 61.20 & 144.00 & 210 & 22.71 & 24.35 \\
\hline & & $30-70$ & 8.10 & 3.51 & - & 2.00 & 15.00 & 20.60 & 15.60 & 6.20 & 15.00 & 0.80 & 4.54 & 5.16 \\
\hline
\end{tabular}


Table4. Cont.

\begin{tabular}{|c|c|c|c|c|c|c|c|c|c|c|c|c|c|c|}
\hline \multirow{2}{*}{$\begin{array}{l}\text { Physiographic } \\
\text { unit }\end{array}$} & \multirow{3}{*}{$\begin{array}{c}\text { Profile } \\
\text { No. }\end{array}$} & \multirow{3}{*}{$\begin{array}{l}\text { Depth } \\
(\mathrm{cm})\end{array}$} & \multirow{2}{*}{$\mathrm{PH}$} & \multirow{2}{*}{$\begin{array}{c}\mathrm{EC} \\
(\mathrm{dS} / \mathrm{m})\end{array}$} & \multicolumn{4}{|c|}{ Soluble Anions (me/l) } & \multicolumn{4}{|c|}{ Soluble Cations(me/l) } & \multirow[b]{2}{*}{ SAR } & \multirow[b]{2}{*}{ ESP } \\
\hline & & & & & $\mathrm{CO}_{3}^{-}$ & $\mathrm{HCO}_{3}{ }^{-}$ & $\mathrm{Cl}^{-}$ & $\mathrm{SO}_{4} 2^{-}$ & $\mathrm{Ca}^{+2}$ & $\mathrm{Mg}^{+2}$ & $\mathrm{Na}^{+}$ & $\mathrm{K}^{+}$ & & \\
\hline \multirow{19}{*}{ 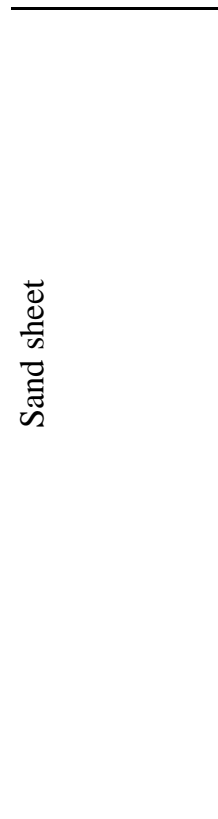 } & & & 8.11 & 9.81 & - & 1.50 & 60.00 & 55.10 & 49.20 & 8.50 & 58.00 & 0.90 & 10.80 & 12.79 \\
\hline & \multirow{3}{*}{16} & $0-30$ & 8.20 & 24.60 & - & 3.00 & 170.00 & 115.00 & 80.50 & 20.20 & 185.60 & 1.70 & 26.18 & 27.19 \\
\hline & & $30-50$ & 8.26 & 15.50 & - & 2.50 & 115.00 & 68.50 & 46.10 & 12.70 & 125.80 & 1.40 & 23.21 & 24.79 \\
\hline & & $50-70$ & 8.28 & 41.80 & - & 1.00 & 420.70 & 70.30 & 30.70 & 19.00 & 427.70 & 4.60 & 85.88 & 55.63 \\
\hline & \multirow{3}{*}{17} & $0-20$ & 7.82 & 22.70 & - & 3.00 & 200.30 & 19.10 & 12.50 & 20.40 & 187.40 & 2.10 & 29.14 & 29.44 \\
\hline & & $20-60$ & 7.55 & 25.60 & - & 2.00 & 225.80 & 79.40 & 70.60 & 33.10 & 201.10 & 2.40 & 27.93 & 28.54 \\
\hline & & $60-90$ & 7.53 & 30.40 & - & 1.50 & 243.30 & 120.00 & 94.30 & 55.70 & 212.20 & 2.60 & 26.20 & 27.12 \\
\hline & \multirow[t]{2}{*}{18} & $0-20$ & 7.91 & 12.10 & - & 3.50 & 109.00 & 37.00 & 22.60 & 13.10 & 112.00 & 1.80 & 26.54 & 27.48 \\
\hline & & $20-60$ & 7.41 & 45.20 & - & 2.50 & 347.50 & 235.60 & 205.00 & 40.00 & 336.00 & 3.10 & 30.44 & 30.38 \\
\hline & \multirow[t]{2}{*}{19} & $0-20$ & 7.60 & 7.83 & - & 3.00 & 32.00 & 50.57 & 43.20 & 7.76 & 34.00 & 0.61 & 6.73 & 9.03 \\
\hline & & $20-40$ & 8.12 & 15.63 & - & 2.50 & 158.00 & 39.41 & 33.80 & 4.80 & 160.00 & 1.31 & 36.45 & 34.91 \\
\hline & \multirow{3}{*}{24} & $0-30$ & 8.14 & 75.10 & - & 3.50 & 387.00 & 294.70 & 250.00 & 8.80 & 340.00 & 6.20 & 26.11 & 27.14 \\
\hline & & $30-80$ & 7.62 & 7.27 & - & 2.00 & 35.00 & 41.50 & 33.80 & 11.10 & 37.00 & 0.60 & 7.81 & 9.31 \\
\hline & & $80-100$ & 7.53 & 25.10 & - & 1.00 & 16.00 & 140.20 & 124.00 & 41.80 & 132.30 & 3.10 & 14.54 & 16.79 \\
\hline & \multirow[t]{2}{*}{26} & $0-20$ & 7.90 & 21.10 & - & 3.50 & 150.00 & 105.50 & 112.80 & 34.20 & 110.10 & 1.90 & 9.08 & 10.82 \\
\hline & & $20-80$ & 8.00 & 18.10 & - & 2.50 & 165.00 & 48.50 & 56.40 & 26.90 & 130.90 & 180 & 20.29 & 22.28 \\
\hline & \multirow{3}{*}{27} & $0-30$ & 7.92 & 4.62 & - & 3.00 & 21.00 & 23.70 & 18.45 & 9.05 & 20.00 & 0.20 & 5.39 & 6.27 \\
\hline & & $30-80$ & 8.02 & 10.71 & - & 2.50 & 64.00 & 63.70 & 49.00 & 15.10 & 65.00 & 1.10 & 11.48 & 14.48 \\
\hline & & 80-130 & 7.04 & 21.10 & - & 2.00 & 185.00 & 108.30 & 97.40 & 15.80 & 180.00 & 2.00 & 10.96 & 12.67 \\
\hline \multirow{10}{*}{$\begin{array}{l}\frac{\pi}{\frac{\pi}{7}} \\
\frac{0}{0} \\
\text { in }\end{array}$} & \multirow{3}{*}{20} & $0-20$ & 7.12 & 115.00 & - & 3.00 & 608.40 & 768.60 & 640.80 & 170.00 & 559.00 & 10.20 & 39.25 & 36.16 \\
\hline & & $20-40$ & 7.33 & 45.20 & - & 2.00 & 312.70 & 270.30 & 117.80 & 54.60 & 408.70 & 3.90 & 44.40 & 38.91 \\
\hline & & $40-70$ & 7.95 & 39.40 & - & 1.50 & 220.90 & 240.60 & 134.50 & 89.90 & 240.40 & 3.20 & 22.70 & 24.50 \\
\hline & \multirow{4}{*}{21} & $0-20$ & 7.60 & 7.93 & - & 3.50 & 42.00 & 45.33 & 42.10 & 8.12 & 40.00 & 0.61 & 7.98 & 10.53 \\
\hline & & $20-50$ & 7.19 & 165.60 & - & 3.00 & 1982.30 & 159.40 & 130.80 & 74.10 & 193.00 & 9.80 & 14.74 & 16.99 \\
\hline & & $50-70$ & 7.46 & 24.10 & - & 2.00 & 207.50 & 76.20 & 58.80 & 14.70 & 210.00 & 2.20 & 34.65 & 33.82 \\
\hline & & $70-90$ & 7.91 & 14.80 & - & 1.50 & 105.00 & 60.43 & 46.13 & 9.40 & 110.00 & 1.40 & 20.87 & 23.35 \\
\hline & \multirow{3}{*}{23} & $0-10$ & 8.04 & 3.10 & - & 2.50 & 14.00 & 16.26 & 15.10 & 4.40 & 13.00 & 0.26 & 4.17 & 4.66 \\
\hline & & $10-25$ & 7.68 & 44.60 & - & 2.00 & 517.60 & 50.80 & 38.10 & 32.46 & 495.8 & 4.10 & 83.61 & 54.97 \\
\hline & & $25-50$ & 7.11 & 189.50 & - & 1.00 & 2084.00 & 300.40 & 270.70 & 503.50 & 1600.00 & 11.90 & 81.34 & 54.28 \\
\hline
\end{tabular}


Table 4 Cont.

\begin{tabular}{|c|c|c|c|c|c|c|c|c|c|c|c|c|c|c|}
\hline \multirow{16}{*}{ 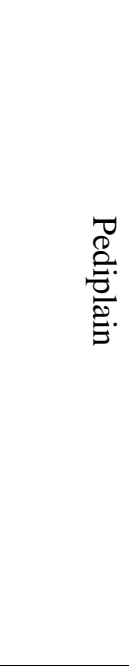 } & 6 & $0-20$ & 7.08 & 9.53 & - & 3.00 & 36.00 & 68.00 & 43.50 & 23.40 & 39.00 & 1.10 & 6.74 & 7.99 \\
\hline & & $20-100$ & 8.04 & 5.83 & - & 1.50 & 43.00 & 15.60 & 12.40 & 4.20 & 43.00 & 0.50 & 14.93 & 17.19 \\
\hline & \multirow{3}{*}{8} & $0-15$ & 8.23 & 78.20 & - & 3.00 & 130.50 & 87.90 & 61.50 & 36.50 & 118.30 & 2.10 & 16.90 & 18.34 \\
\hline & & $15-30$ & 7.68 & 2.90 & - & 2.00 & 16.00 & 11.90 & 10.20 & 4.50 & 15.00 & 2.00 & 7.14 & 8.48 \\
\hline & & $30-40$ & 7.60 & 16.40 & - & 1.00 & 67.00 & 128.80 & 113.60 & 20.50 & 61.00 & 1.70 & 7.40 & 8.87 \\
\hline & 9 & $0-40$ & 7.11 & 7.01 & - & 3.00 & 36.00 & 35.60 & 30.80 & 6.10 & 37.00 & 0.70 & 8.62 & 10.27 \\
\hline & \multirow{3}{*}{13} & $0-15$ & 7.42 & 11.25 & - & 3.00 & 50.00 & 82.94 & 56.13 & 24.90 & 54.00 & 0.91 & 8.45 & 10.12 \\
\hline & & $15-30$ & 7.65 & 18.10 & - & 2.05 & 152.50 & 70.71 & 61.50 & 22.00 & 140.00 & 1.70 & 21.63 & 24.19 \\
\hline & & $30-50$ & 7.83 & 13.80 & - & 1.50 & 125.00 & 43.50 & 35.90 & 11.20 & 120.00 & 2.90 & 24.74 & 26.73 \\
\hline & \multirow[t]{2}{*}{14} & $0-15$ & 7.47 & 11.90 & - & 3.00 & 76.00 & 51.90 & 41.00 & 11.90 & 77.00 & 1.00 & 14.98 & 17.24 \\
\hline & & $15-60$ & 7.95 & 101.60 & - & 2.00 & 638.80 & 578.40 & 491.10 & 109.50 & 608.50 & 11.10 & 35.15 & 33.59 \\
\hline & \multirow{3}{*}{22} & $0-15$ & 8.08 & 16.50 & - & 3.00 & 155.00 & 42.00 & 56.40 & 17.10 & 125.30 & 1.20 & 20.67 & 22.62 \\
\hline & & $15-30$ & 7.85 & 7.90 & - & 2.50 & 60.00 & 30.24 & 25.20 & 9.10 & 58.00 & 0.44 & 14.01 & 16.25 \\
\hline & & $30-40$ & 7.65 & 20.30 & - & 1.50 & 157.00 & 123.40 & 87.10 & 32.90 & 160.00 & 1.90 & 20.67 & 22.62 \\
\hline & \multirow[t]{2}{*}{25} & $0-35$ & 8.06 & 51.30 & - & 2.00 & 480.30 & 180.70 & 152.80 & 55.10 & 450.30 & 4.80 & 44.15 & 39.41 \\
\hline & & $35-70$ & 7.64 & 36.10 & - & 1.50 & 238.80 & 227.70 & 215.80 & 25.20 & 223.80 & 3.20 & 20.41 & 23.14 \\
\hline \multirow{2}{*}{ Dune } & 28 & $0-100$ & 8.1 & 1.7 & - & 0.8 & 13.2 & 4.1 & 3.8 & 2.2 & 11.9 & 0.2 & 6.87 & 8.15 \\
\hline & 29 & $0-100$ & 8.2 & 1.4 & - & 0.6 & 11.7 & 2.8 & 2.6 & 1.8 & 10.5 & 0.2 & 7.08 & 8.41 \\
\hline
\end{tabular}


Table 5. Evaluation of the studied soils according to Sys \&Verheye(1978)

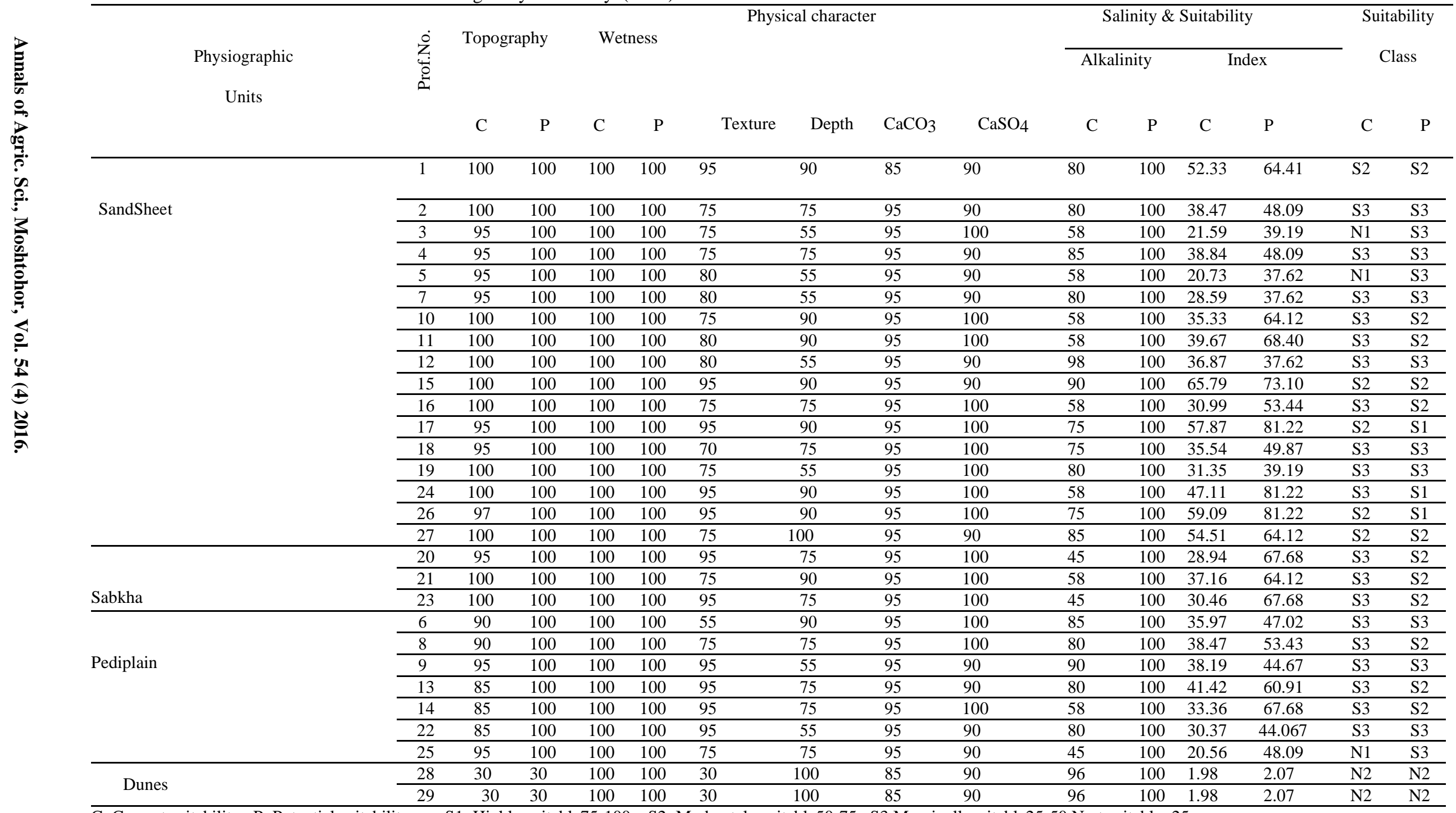




\section{References}

Abdel Kawy WA, and Kh. M .Darwish (2014), Assessment of optimum land use and water requirements for agricultural purpose in some soils, South Paris Oasis, Western Desert, Egypt, Arab J Geoci DOI 10.1007/s12517-013-1054-7.

AtlasofEgypt(1928), Fouad in Cairo University, Cairo.

Black, C.A.(1965), "Methods of Soil Analysis". Amer. Soc. Agron. Inc. publisher. USA.

EGSA(Egyptian Geology Survey Authority) (1988), Egyptian General petroleum Corporation: Geological Map of Egypt", Conoco Coral, printed in Germany by institute fur Angewandt Geodasie, Berlin, Technisc Fashhochschule Berlin, 1988, Scale 1:500000.

FAO/SF: 16/UAR(1963), High Dam Soil Survey, United aAab Republic Volume II, the Reconnaissance Soil Survey, United Nations Development Porgram (Special Fund).

FAO (1994), "Guidelines For Soil Profile Description" .Third Edition, Rome, Italy.

Goosen, M.L.(1967), "Aerial photo-interpretation in Soil Survey" .Soil Bull.No.6,FAO,Rome,Italy.

Jackson, M.L.(1958), "Soil Chemical Analysis". Prentie- Hall Inc. Englewood Cliffs., N.J.,UK.

Jackson, M. L. (1975),"Soil Chemical Aanalysis". Advanced course. Soil Sci. Dept., Mas., Wisc., USA
Lueder, D. R (1959), "Aerial Photographic Interpretation". Principles and Applications. Mc Grow-Hill Book Company Inc. New York, USA.

Egyption Meteorological Authority (2014), "The Normal for Egypt up to 2014. Ministry of Civil Aviation, Cairo, Egypt. Mete0orological Res. Bull.,32:55-65.

Piper,C.S.(1950), "Soil and plant analysis Int. Scipublishers: Inc New Yourk, USA.

Richard,L.A.(1954), "Diagnosis Improvement of Saline and Alkali Soils". U. S. Dept. Agric. Hand book No. 60, Washington, DC,USA.

Robert L. B. and m Julia A. J (1983),"Dictionary of Geological Terms". Doubleday, New York, USA.

Sabins, F.F (1978),"Remote Sensing Principles and Interpretation". Freeman\& Co. San Fransisco, USA.

Sys.C. and W Verheye(1978), An attempt to the evaluation of physical land characteristics for irrigation according to the FAO framework for land evaluation. Int.Train.Center for PostGrad.,Soil.Sci,.,Ghent,Belgium.

USDA (2014), key of Soil Taxonomy. Prepared by Agronomy Department Cornelluni. Ithaco, NewYork,USA.

Vink, A.P.A (1963), "Aerial Photographs and the Soil Sciences". UNESCO, Proc. Toulouse Conf. Canada 


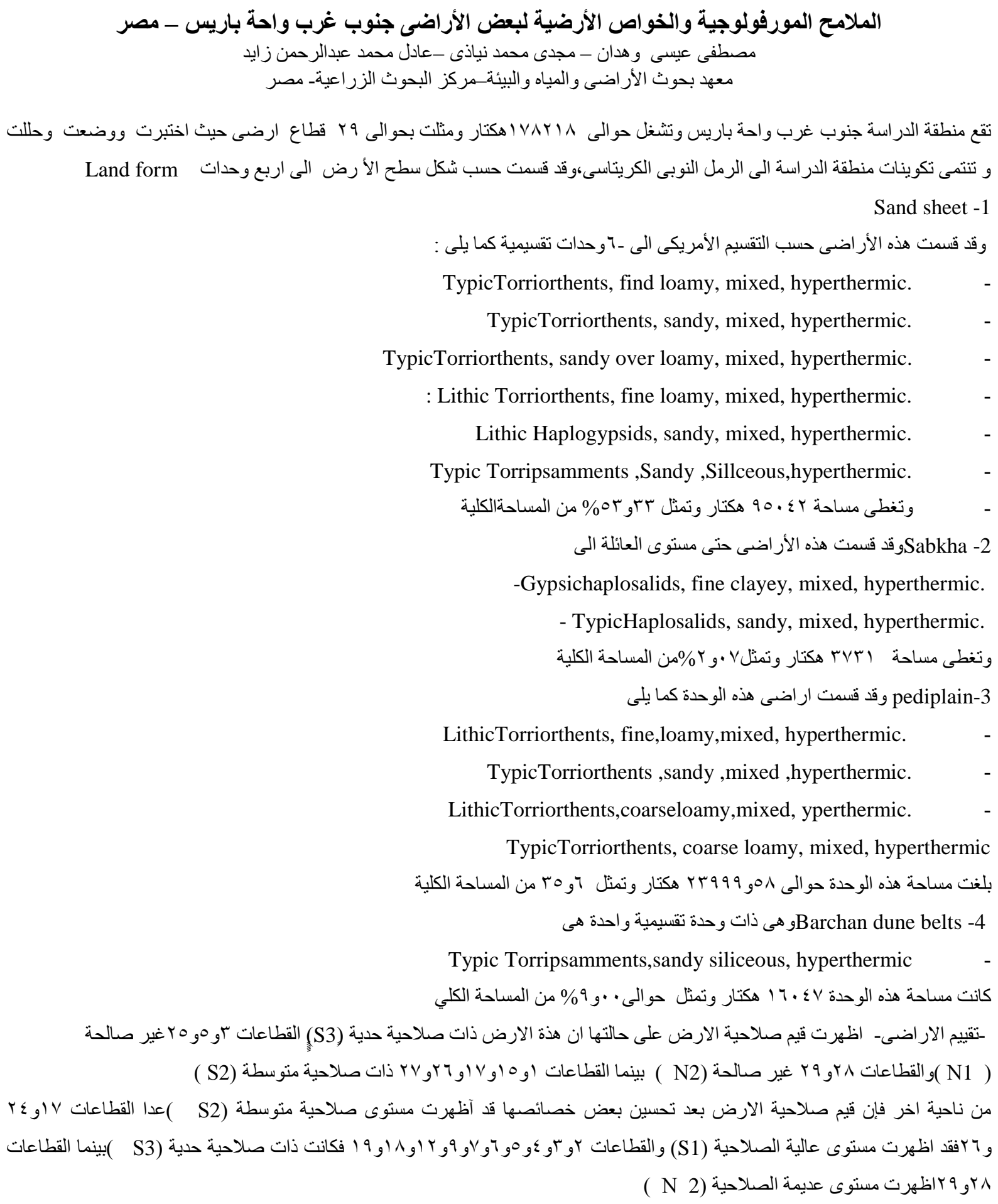

-Gypsichaplosalids, fine clayey, mixed, hyperthermic.

- TypicHaplosalids, sandy, mixed, hyperthermic.

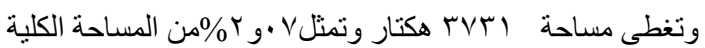
pediplain-3

LithicTorriorthents, fine,loamy,mixed, hyperthermic.

TypicTorriorthents ,sandy ,mixed ,hyperthermic.

LithicTorriorthents, coarseloamy,mixed, yperthermic.

TypicTorriorthents, coarse loamy, mixed, hyperthermic

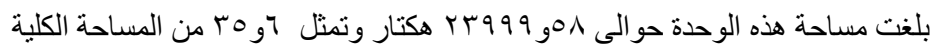
Barchan dune belts -4 Typic Torripsamments,sandy siliceous, hyperthermic

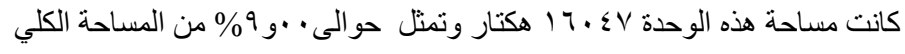

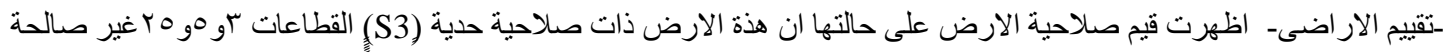

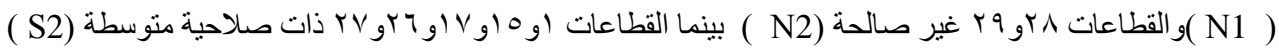

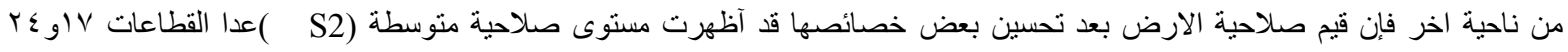

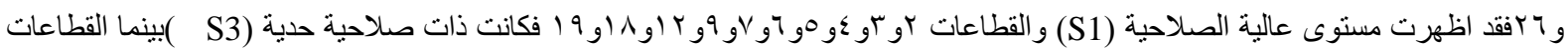

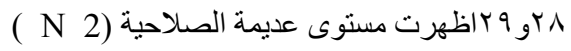

